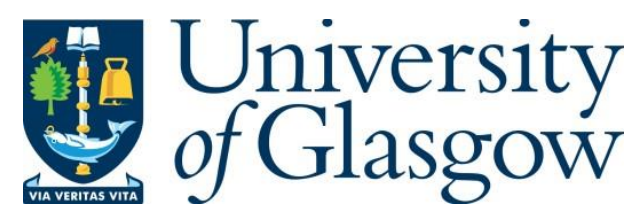

Zielinski, A. T., Kalberer, M., Jones, R. L., Prasad, A. and Seshia, A. A. (2016)

Particulate Mass Sensing With Piezoelectric Bulk Acoustic Mode Resonators. In: 2016 IEEE International Frequency Control Symposium (IFCS), New Orleans, LA, USA, 0912 May 2016, ISBN 9781509020911.

There may be differences between this version and the published version. You are advised to consult the publisher's version if you wish to cite from it.

http://eprints.gla.ac.uk/204443/

Deposited on: 2 December 2019

Enlighten - Research publications by members of the University of Glasgow http://eprints.gla.ac.uk 


\section{Particulate Mass Sensing with Piezoelectric Bulk Acoustic Mode Resonators}

\author{
Arthur T. Zielinski, Markus Kalberer, \\ Roderic L. Jones \\ Department of Chemistry \\ University of Cambridge \\ Cambridge, UK \\ arthur.zielinski@atm.ch.cam.ac.uk
}

\author{
Abhinav Prasad, Ashwin A. Seshia \\ Department of Engineering \\ University of Cambridge \\ Cambridge, UK
}

\begin{abstract}
Current portable particle detection instruments typically rely on optical methods which are limited to $100 \mathrm{~nm}$ diameter particles. Microfabricated bulk acoustic resonators, when used as mass balances, could take particle detection below this limit. This study examines the collection of particles onto piezoelectric bulk acoustic mode resonators from gaseous flow using classical impaction. Collection of both polystyrene latex particles and $\alpha$-pinene secondary organic aerosol particles was examined in terms of frequency shift and collection efficiency. A new experimental setup was introduced which allows for adjusting major impactor, resonator, and aerosol properties. Preliminary results show the setup works for both particles while the saturation limit was not reached within an hour despite highly elevated particle concentrations.
\end{abstract}

Keywords-MEMS resonators; particle collection; impactor; piezoelectric; square extensional mode

\section{INTRODUCTION}

There is a growing understanding of the detrimental effects of atmospheric particles on both the environment [1] and human health [2]. In order to monitor ambient particle concentrations several small-scale, portable instruments have been produced with recent designs being primarily based on optical methods that are limited to detecting particles with diameters larger than $100 \mathrm{~nm}$ [3]. Recent studies have shown that sub-100 nm particles may be responsible for most adverse health effects [2] meaning a new sensing method suitable for small sensor integration is desirable.

A possible solution exists using Microelectromechanical systems (MEMS) resonators acting as high sensitivity mass balances. This approach has the additional benefit that the mass is the measured quantity unlike optical approaches which infer the mass based on assumed densities and particle morphology. Depending on the application, different resonator topologies and particulate collection methods have been studied. Paprotny et al. [4] detected particles with thin-film bulk acoustic resonators collected via thermophoresis while Mehdizadeh et al. [5] combined thermally actuated, piezoresistively sensed resonators with inertial impaction. For beam-type resonators, Schmid et al. [6] used nanomechanical resonant filter fibre (doubly-clamped beams) with impaction and minor Brownian diffusion collection while Wasisto et al. [7] used a piezoresistive silicon cantilever to measure particles collected electrostatically.
Each study removed undesirable, larger particles from the air flow using impactors [4, 5], impactors and filters [7] or by producing specific particle distributions [6]. Using impactors allows for size speciation when arranged in a series (i.e. a cascade) arrangement as demonstrated by Mehdizadeh et al. [5] which provides additional information about the sampled particle distribution. Furthermore, impactors, when used for collection, minimally influence the particles in question whereas thermophoresis and electrostatic collection both change physical properties (temperature and charge, respectively).

In this work, piezoelectric bulk acoustic mode resonators are employed as impaction plates in a newly designed MEMS Impactor Stage (MIS). The experimental setup allows for a wide range of test protocols due to its modular design. Initial results focus on the collection of submicron particles produced in a laboratory setting. Two varieties of particle were tested, polystyrene latex (PSL) particles and $\alpha$-pinene secondary organic aerosol, to highlight the versatility of the experimental setup to vary input conditions and verify the functionality of the experimental setup.

\section{MATERIALS AND METHODS}

\section{A. Flow system and particle production}

The flow system used to measure particles is shown in Fig. 1 which used two configurations (not pictured) for aerosol production discussed below. The MIS, a subset of the overall system, is described in detail in the following subsection.

Two particle varieties were used during this study: $296 \pm 6 \mathrm{~nm}$ polystyrene latex (PSL) particles (Nanosphere Size Standards, Thermo Fisher Scientific) and $\alpha$-pinene secondary organic aerosol (SOA). PSL particles are known for their spherical nature while $\alpha$-pinene SOA are also commonly spherical [8]. PSL particles are typically used as calibration standards due to their narrow size distribution. They are solid particles that are expected to have relatively high particle bounce-off. In this study, PSL particles are nebulised with dry nitrogen gas from suspension in High Performance Liquid Chromatography (HPLC) grade water and dried through a diffusion dryer. The SOA formed from $\alpha$-pinene ozonolysis is a commonly studied, atmospherically relevant aerosol particle. Production of the SOA was done using an ozone lamp with multiple emission wavelengths (primarily $184.9 \mathrm{~nm}$ ) which 
converted oxygen from synthetic air to ozone before mixing with $\alpha$-pinene vapours. Before being size selected, the SOA passed through a charcoal denuder to remove any volatile gases from the flow that may affect downstream equipment. The resulting SOA viscosity is highly dependent on the ambient conditions (e.g. relative humidity and age [9]). The difference in viscosity between PSL and $\alpha$-pinene SOA should be apparent in terms of their collection efficiency due to the implied particle bounce-off differences [10].

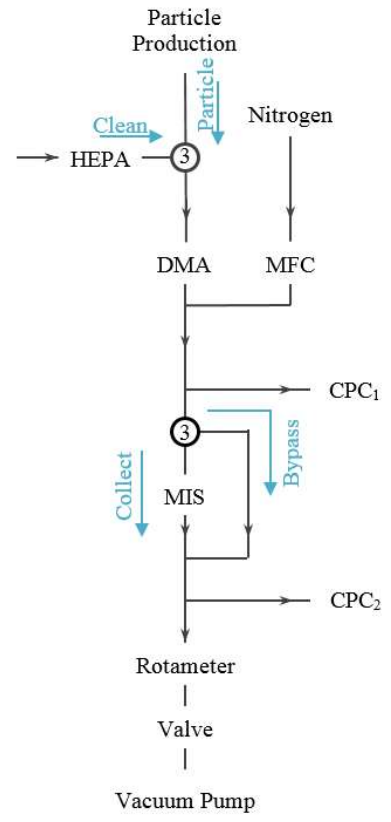

Fig. 1. Schematic of main flow measurement components neglecting particle production. Blue arrows labelled "Clean", "Particle", "Collect", and "Bypass" denote the pathways that the two three-way valves control. "Clean" brings ambient air through a HEPA filter which should be particle-free whereas "Particle" allows for particles from the upstream production to be introduced to the system. "Bypass" avoids the resonator and the MIS entirely while "Collect" directs flow through the nozzle and at the resonator. Flow rates are given in text. Acronymns: HEPA $\equiv$ "High-Efficiency Particulate Arrestance", DMA $\equiv$ "Differential Mobility Analyser", MFC $\equiv$ "Mass Flow Controller", $\mathrm{CPC} \equiv$ "Condensation Particle Counter", MIS $\equiv$ "MEMS Impactor Stage".

Once either particle is produced, it flows towards a differential mobility analyser (DMA) for size-selection based on its electric mobility. The DMA (Model 3081, TSI) was set to allow $\sim 296 \mathrm{~nm}$ particles through during all tests. In order to maintain a sheath-to-sample flow ratio of 10-to-1 as recommended for highest efficiency, the flow through the DMA was controlled to be approximately 1.0 LPM to ensure that $296 \mathrm{~nm}$ particles could be size selected.

External reference particle measurement was done by two condensation particle counters (CPCs) upstream (Model 3776, TSI) and downstream (Model 3776, TSI) of the MIS. The flow through each CPC is controlled by a critical orifice limiting flow to $0.3 \mathrm{LPM}$. The CPCs also provide pressure measurements for the incoming flow. CPC particle measurements have a per second resolution.

The final flow control, a vacuum pump, is adjusted using a needle valve to maintain 0.7 LPM draw as measured by a rotameter and confirmed by a flow measurement by the DMA.

\section{B. MEMS Impactor Stage (MIS)}

The MIS is a highly-modular single stage impactor designed for collecting particles on MEMS resonators. The main components are the enclosure, the nozzle, and the transduction printed circuit board (PCB) that holds the chip.

The enclosure houses ports for signal transduction (four SMA ports), temperature and RH measurements (SHT21, Sensirion) as well as the nozzle inlet and $1 / 4$ " Swagelok outlet. The nozzle consists of an $1 / 8$ " stainless steel tube with a black acetal insert. Following classic inertial impaction, the nozzle is used to accelerate particles towards the impaction surface, a resonator, and collect particles based on their inertia. Larger particles are collected while smaller particles stay within the particle flow. In this study, the insert had $3 \times 0.25 \mathrm{~mm}$ jets (although this is easily replaceable) such that, under 1.0 LPM flow conditions, it is expected to have a cut-off diameter (i.e. the diameter at which $50 \%$ of particles are collected) of approximately $280 \mathrm{~nm}$. This design, based on the principles introduced by Marple and Willeke [11], neglects compressibility effects so the precise cut-off diameter requires empirical verification. Experimental particle collection was conducted at 0.7 LPM flow to limit the pressure drop across the MIS so lower empirical collection efficiencies were expected.

The translation stage, which holds the transduction PCB, allows for two-axis nozzle alignment in the plane of the chip. The nozzle holder is used to adjust the nozzle-to-plate distance. Nozzle alignment with the translation stage is done with an alternative attachment replacing the nozzle with a USB microscope (VMS-001, Veho) assuming concentricity between the two attachments. The PCB allows for any chip to be implemented for the specified chip carrier (LCC04420, Spectrum Semiconductor Materials, Inc.) as it provides SMA connections for each contact pad. The PCB combined with the translation stage allows for any resonator on a given chip to be targeted for collection.

\section{Resonators and signal processing}

The resonators used in this study are square $1400 \mu \mathrm{m}$ side length piezoelectric bulk acoustic mode resonators with four triangular electrodes enabling detection of multiple modes. The electrodes are a patterned stack of aluminium and aluminium nitride on the base single-crystal silicon. For further details on the resonator design, see Prasad et al. [12]. The square extensional mode was the focus of this study, despite its known spatial sensitivity [13], due to its wide application in many resonator topologies and higher amplitudes with the specific topology. The Lamé and Butterfly modes were electrically suppressed following configuration 3 [12]. Table 1 shows the dimensions and additional details of the resonators.

The resonators were transduced in a two-port configuration (i.e. actuating and sensing from two separate sets of electrodes) with the resonator body grounded to limit parallel feedthrough capacitance. The devices were tested was open-loop with a network analyser (N9915A, Agilent) to extract transmission parameters. 
TABLE 1. RESONATOR DIMENSIONS AND SENSING PARAMETERS

\begin{tabular}{lcl}
\hline Parameter & Unit & Value \\
\hline Resonator side length & $\mu \mathrm{m}$ & 1400 \\
Maximum thickness & $\mu \mathrm{m}$ & 11.5 \\
Natrual frequency & $\mathrm{MHz}$ & $\sim 3.16$ \\
Span & $\mathrm{kHz}$ & 20 \\
Frequency resolution & $\mathrm{Hz}$ & \pm 25 \\
AC Power & $\mathrm{dBm}$ & $0^{\mathrm{a}}$ \\
Mode & - & Square Extensional \\
\hline \multicolumn{2}{|}{}
\end{tabular}

\section{General test procedure}

Three resonators were used for experimentation with two collecting PSL particles and one collecting $\alpha$-pinene SOA. Collection of particles was on the back side of the resonators to protect the electrodes and wire bonds from particles and air flow.

The test procedure, repeated for each resonator, began with the insertion, alignment, and characterisation of the unloaded resonator. Each resonator went through five flow phases: (1) initial particle counting bypassing the MIS, (2) running clean air through the bypass, (3) pressurising the MIS with clean air, (4) collecting particles on to the resonator, and (5) repeating the particle counting while bypassing the MIS. These phases were controlled primarily by adjusting the two three-way valves in the system shown in Fig. 1. Particles were produced throughout the experiments and sent to an exhaust line when not being counted and/or collected. The collection period was one hour long with frequency data being logged at minute intervals. A final frequency measurement was taken after the test was complete and the MIS was unsealed.

Images of the resonator were taken pre- and post-collection with the USB microscope. More detailed images of the collection patterns were also taken once the resonator was removed from the MIS using an optical microscope (BX51, Olympus).

\section{RESULTS}

\section{A. Studies with solid PSL particles}

The resonator output data for the two PSL collection experiments are shown in Fig. 2. No quality factor (Q-factor) information is given for the first resonator due to non-linear effects observed during most of the experiment. It can be seen that during the first two stages, when the MIS (i.e. the resonator) is being bypassed, there are no changes in frequency or Q-factor. When the clean air enters the MIS (stage 3), there is a slight shift in resonant frequency and Q-factor. Once collection begins, a clear negative shift begins on the $\mathrm{kHz}$ scale over one hour. The shifts in frequency are non-linear, with their slope gradually increasing in magnitude which can be explained by the particle distributions being collected.

The two resonators were exposed to different particle concentrations which should ideally be constant assuming the nebuliser output stabilises, but in reality an increasing particle concentration was recorded throughout both collection periods. The input concentrations for the tests reached maximums on the order of 4500 particles/cc and 3125 particles/cc, respectively. The difference between upstream and downstream particle concentrations were integrated and converted to a mass based on the flow rate and particle size as shown in Fig. 3. The difference in CPC measurements was assumed to be a measure of the number of particles that should be collected by the resonator. In reality, there are particle losses including diffusion (i.e. wall losses), coagulation, and collection elsewhere. Furthermore, a slight time delay exists between CPCs due to the residence time of the MIS which is relevant for varying particle concentrations.
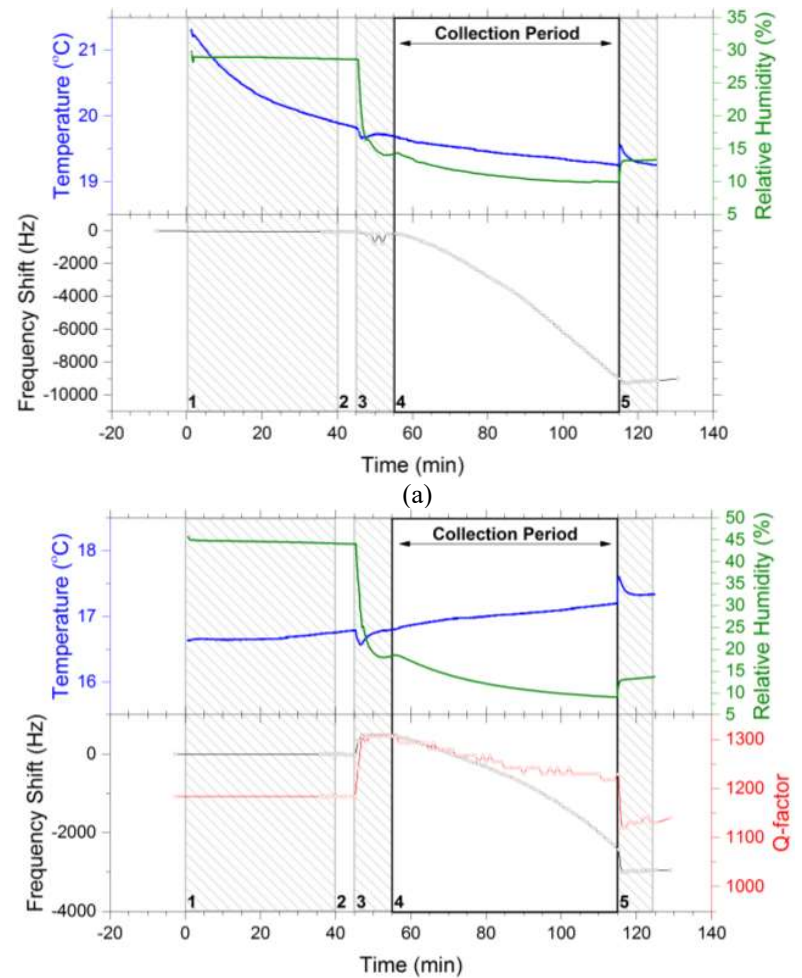

(b)

Fig. 2. Relative frequency shift data for the (a) first and (b) second PSL collection tests (black) with quality factor (red), temperature (blue), and relative humidity (green) traces. The first test has no Q-factor information due to non-linearities observed in the frequency response. The collection phase, phase 4 , is bolded to highlight the frequency stage. The remaining phases are as described in Section II.D.

The recorded frequency shifts were used to estimate mass using an average mass sensitivity based on Sauerbrey's equation [13] neglecting stiffness changes. The temperature correction factor, roughly $-35 \mathrm{ppm} /{ }^{\circ} \mathrm{C}[12]$, was neglected as its influence is an order of magnitude lower than the observed shifts as temperature changes were less than $1{ }^{\circ} \mathrm{C}$ during collection phases. The resulting mass estimations were plotted alongside the CPC derived masses in Fig. 3 after being scaled to the level of the CPC masses. The scaling factors for the tests were 5.7 and 9.2, respectively, based on correlation plots with $r^{2}$ values greater than 0.99 . While these factors account for the particle losses discussed above, they also account for the degree of resonator coverage in terms of its spatial sensitivity. The second test having a larger scaling factor implies the collected mass, while lower in total amount, was collected in more sensitive areas of the resonator (see Fig. 6 for comparison and discussion below). Once scaled, the two estimations track one another highlighting that the changing particle 
concentrations are registered by the resonators based on the changing rates of frequency shifts seen in Fig. 2.

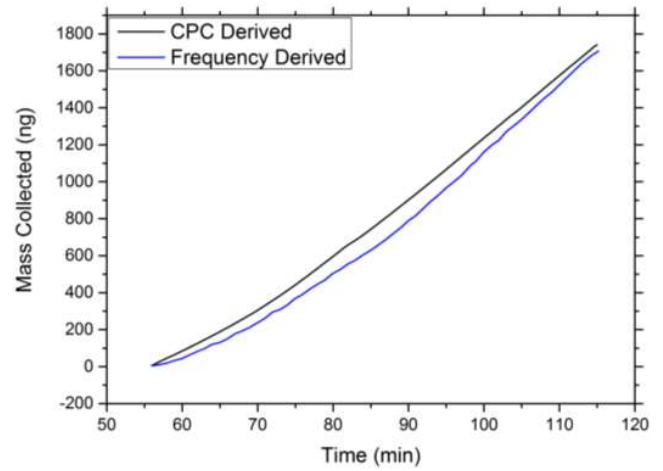

(a)

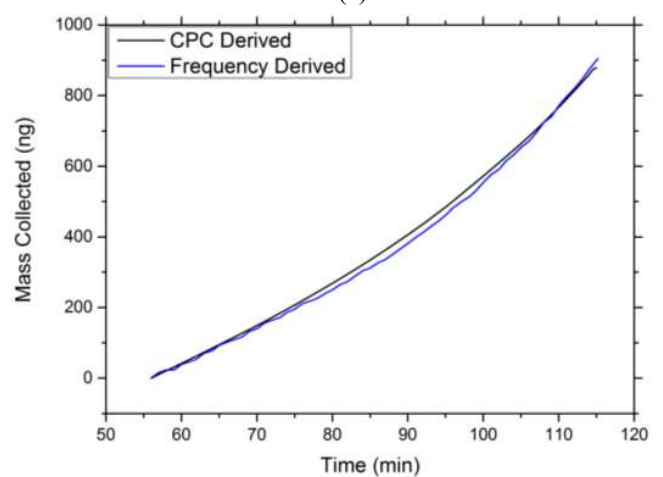

(b)

Fig. 3. Estimated mass during (a) first and (b) second PSL collections based on integrated CPC difference (black) and frequency shifts (blue). CPC derived estimation assumes all particles removed from flow are collected on resonator. The frequency derived estimation assumes a universal mass sensitivity and has been scaled to CPC derived mass levels. Scaling factors were 5.7 and 9.2, respectively. Estimations show strong correlation once linearly scaled. Using a theoretical sensitivity of $-29.5 \mathrm{~Hz} / \mathrm{ng}$ and assuming all particles are $296 \mathrm{~nm}$. CPC data initially averaged with a two-minute moving average and the mass was zeroed after 1 minute of collection to reduce the influence of pressure fluctuations and residence time.

\section{B. Comparison with $\alpha$-pinene $S O A$}

The output of the resonator during SOA collection is shown in Fig. 4 with corresponding temperature and humidity data. Similar to the PSL experiments, the resonator was stable until particle collection began except for a minor shift when clean flow was introduced to the resonator. The resonator saw a much larger total frequency shift corresponding to a higher particle concentration (roughly $35000 / \mathrm{cc}$ ). The quality factor fluctuated which may be due to several factors (e.g. flow rate, relative humidity) in addition to particle adsorption. The particle mass estimated by the CPCs and the resonator are shown in Fig. 5, similarly to the PSL tests, with two slight differences. Firstly, the particle density is difficult to define for $\alpha$-pinene SOA, so a value of $1000 \mathrm{~kg} / \mathrm{m}^{3}$ was used based on literature estimates [8]. Secondly, ozonolysis produces a stable output once the ozone lamp stabilises meaning the frequency shifts are linear which is confirmed by both mass estimates. The correlation-based scaling factor used between the CPC and frequency estimations was larger for the $\alpha$-pinene SOA at $10.0\left(r^{2}>0.99\right)$ which may be influenced by particle morphology, but it is difficult to decouple from concentration and particle loss effects.

The difference in particle morphology is clear from the optical images shown in Fig. 6 of the resonator collection surface. Both PSL collections show conglomerations of solid particles based on the nozzle positioning with one of the three jets appearing clogged. The difference in particle concentration is clear between Fig. 6(a) and (b) in the final collection density, with the slight alignment differences partially explaining the different scaling factors. Since the square-extensional mode has a node at the centre and antinodes at the four corners, the collection pattern for Fig. 6(b) is in a more sensitive area than Fig. 6(a). In contrast to PSL, the $\alpha$-pinene collection (Fig. 6(c)) shows a thin fluid film (highlighted by interference fringes) on the resonator surface with lessening degrees of coalescence away from the jets. The collected particles are likely to be highly viscous, in agreement with expectations, as the nozzle pattern is still clear despite the image being taken the day after collection. It appears the previously clogged jet has unclogged based on the additional large collection along the left edge of the resonator.

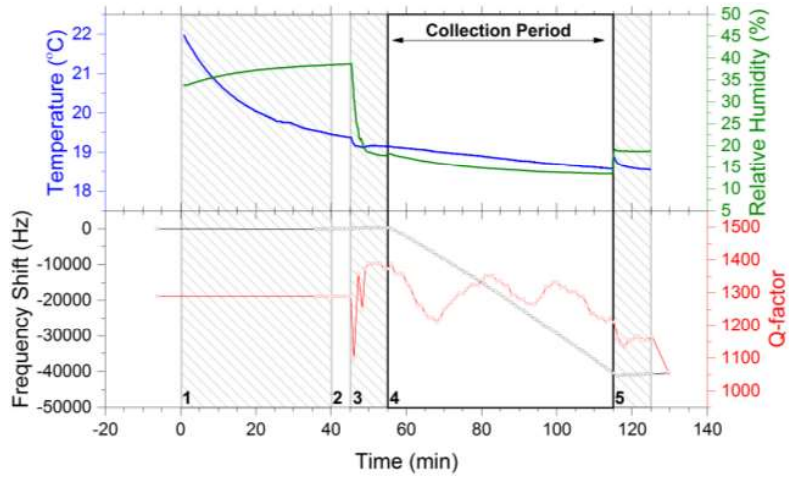

Fig. 4. Relative frequency shift data for $\alpha$-pinene collection (black) with quality factor (red), temperature (blue), and relative humidity (green) traces. The collection phase, phase 4 , is bolded to highlight the frequency stage. The remaining phases are as described in Section II.D.

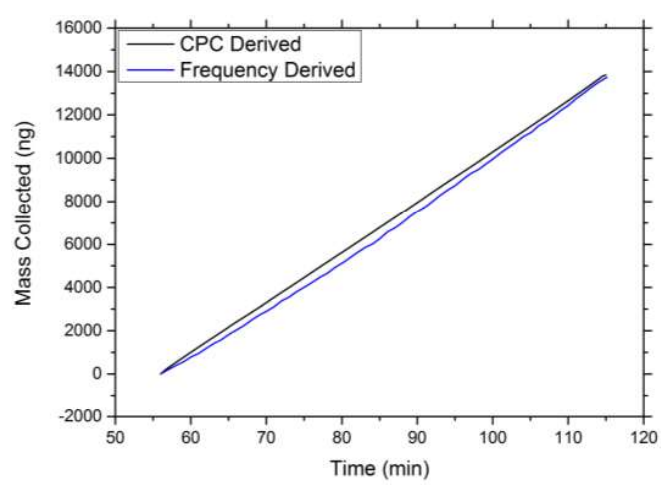

Fig. 5. Estimated mass during $\alpha$-pinene SOA collection based on integrated CPC difference (black) and frequency shifts (blue). CPC derived estimation assumes all particles removed from flow are collected on resonator. The frequency derived estimation assumes a universal mass sensitivity and has been scaled to CPC derived mass levels by a factor of 10.0. Estimations show strong correlation once linearly scaled. Using a theoretical sensitivity of -29.5 $\mathrm{Hz} / \mathrm{ng}$ and assuming all particles are $296 \mathrm{~nm}$. CPC data initially averaged with a two-minute moving average and the mass was zeroed after 1 minute of collection to reduce the influence of pressure fluctuations and residence time. 


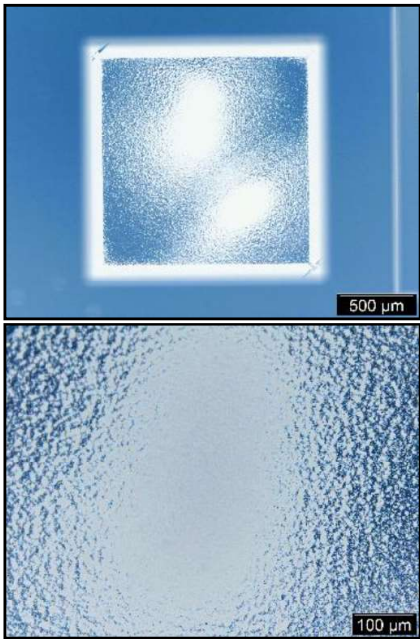

(a)

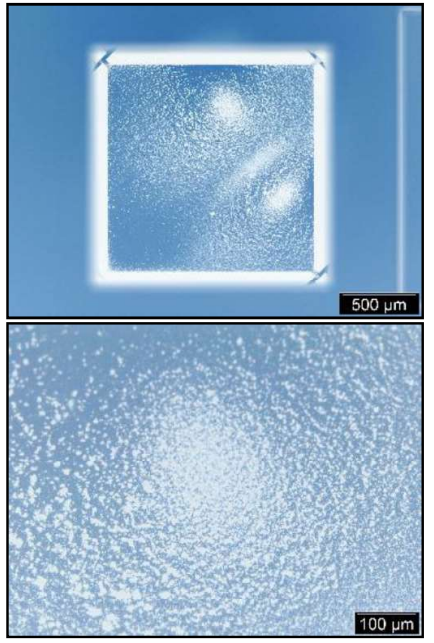

(b)

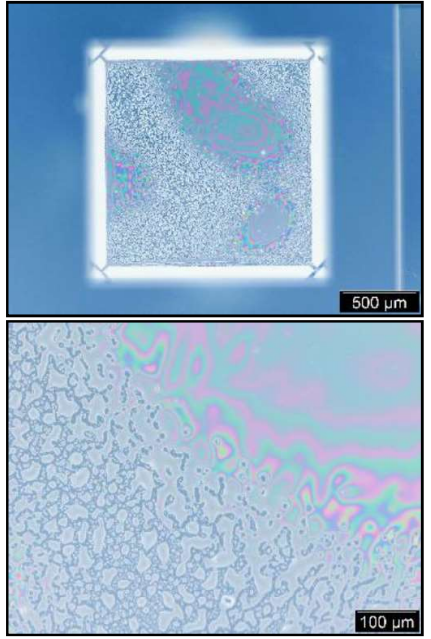

(c)

Fig. 6. Images of the full resonator (top) and detailed collection areas (bottom) for the (a) first and (b) second PSL experiments compared to (c) the $\alpha$-pinene SOA test. PSL particles appear white solids in the images while $\alpha$-pinene SOA appears as a thin film showing interference fringes. The particle collection pattern is clear showing two nozzle collection spots (areas with higher particle density) for PSL collection but potentially three for $\alpha$-pinene collection.

The resonators reacted well to both particle varieties but a direct comparison remains difficult without accounting for the influence of particle concentration and spatial sensitivity (i.e. nozzle alignment). Possible differences include solid particles not spreading across the resonator as efficiently and, for all particles, higher concentrations likely increase the likelihood of particle losses due to the impactor (e.g. particle re-entrainment) and the flow itself (e.g. particle coagulation). Furthermore, as particles become more liquid-like, they will be more likely to adhere to the resonator edges rather than being pushed off due to the surface tension as highlighted in Fig. 7.

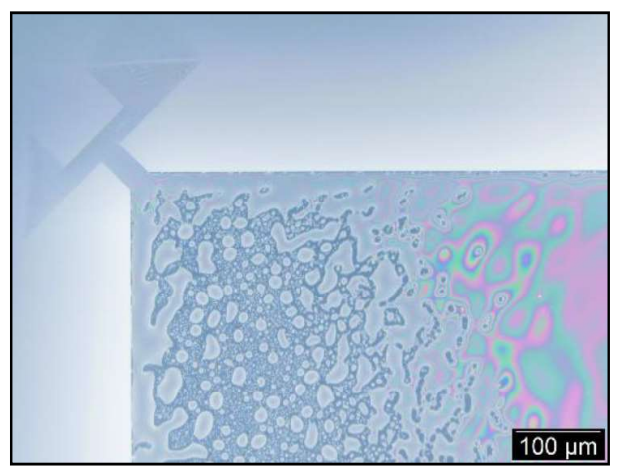

Fig. 7. Corner of $\alpha$-pinene collection resonator showing surface tension effects along edge of resonator.

A key interest for applying microresonators as mass sensors is their saturation time. The expected mechanism for saturation is the energy dissipation (i.e. attenuation of the shear wave) within the adsorbed medium as it collects until a point where the energy is unable to penetrate additional mass. Such a mechanism is viscosity dependant and widely seen in hydrodynamic MEMS applications [14]. At the concentrations discussed during this study $(4500,3125$, and 35000 particles/cc), saturation was not apparent after an hour of continuous sampling at 0.7 LPM. In comparison, Diapouli et al. found 24-hour mean concentrations of all particles sized
$100-3000 \mathrm{~nm}$ on the order of $3 \times 10^{3}$ particles/cc. [15] in Athens, Greece. A multiple hour saturation time would then be expected, for $296 \mathrm{~nm}$ particles, but is highly dependent on particle diameter. Saturation time can be extended by reducing the flow rates involved with the added benefit of reducing the required pump power. Experimentally larger particles or longer collection periods are required to determine saturation limits.

\section{Effectiveness of MIS}

The collection efficiency of an impactor is a measure of the percentage of particles that have been captured by the impactor. Its value is primarily based on the nozzle and flow parameters as discussed above but can be influenced by particle interactions with the impaction surface (i.e. particle bounce-off). Solid particles are therefore expected to have lower collection efficiencies than viscous particles. In this study, the collection efficiencies were estimated based on the ratio of particle concentrations at the inlet and outlet of the MIS (based on CPC readings) and is a measure of the particle collection in the MIS as a whole rather than the resonator itself. Empirical collection efficiencies of the PSL particles were $74 \%$ and $75 \%$, respectively, while the $\alpha$-pinene SOA collection efficiency was unexpectedly lower at $72 \%$. While all three collection efficiencies are relatively high despite the lowered flow rate from the initial design (0.7 LPM vs. 1.0 LPM), it is unlikely all particles lost can be associated with collection on the resonator as previously discussed with Fig. 3 and Fig. 5. The SOA collection efficiency being lower could be due to the nozzle being partially unclogged meaning the flow velocity through each jet was lower thus lowering the collection efficiency. It could also simply be an issue due to the high particle concentrations unrelated to particle type or the nozzle. The collection efficiency of all resonators might be improved by adding a silicone oil to the resonator collection surface which is common practice with filter based impaction [11]. Due to the substantial frequency shifts seen during the 
current study, such a layer may not decrease the resonator lifetime beyond a useable limit while increasing particle detection. The collection efficiency of the MIS is independent of the resonator performance, as the resonator will be influenced primarily by nozzle alignment and the measurement mode.

The pressure drops across the MIS, as measured by the CPCs, were on the order of $30-40 \mathrm{kPa}$ despite lowering the flow rate from the original 1.0 LPM which caused even larger pressure drops. While large pressure drops are not of major concern in a laboratory setting, a portable system would have a limited pump size meaning further nozzle considerations may be required.

\section{CONCLUSIONS AND FUTURE WORK}

Microfabricated piezoelectric bulk acoustic mode resonators have been shown to function as effective mass balances under impaction conditions. Despite being exposed to elevated particle concentrations, the studied resonators did not saturate within an hour and correlated well with traditional particle measurements after accounting for a linear offset. Differences in particle type were highlighted optically while quantifying the differences requires uncoupling of the concentration-dependent factors. The experimental impactor, as a whole, had a high collection efficiency on the order of $70 \%$ at $296 \mathrm{~nm}$ for both particle types at a flow rate of 0.7 LPM. The setup was shown to be capable of doing several characterisation-style experiments. The collection efficiency could likely be improved by adding layers of silicone oil, or equivalent, to the collection surface of the resonator without drastically decreasing resonator lifetime.

While the current study showed that the resonators can work for the given application, further studies on the saturation limit and comparisons between different aerosol types is required to understand how well microresonators would work for different applications (e.g. environmental monitoring). A major concern is the small percentage of particle loss in the MIS that can be explained by the frequency shift of the resonators. The difference is likely a combination of particles collecting on areas other than the resonator and analytical shortfalls in the estimation.

The current system showed that it could provide high resolution data that can detect fluctuations in particle production. Problems with nozzle clogging highlight a potential need for a redesign or simple cleaning procedure. The analysis of the data can further be improved by accounting for the spatial and temperature sensitivities of the resonators to account for the slight nozzle misalignments and temperature differences. The CPC data can also be resolved to account for expected losses such as particle coagulation and wall losses due to diffusion.

\section{ACKNOWLEDGEMENTS}

The authors would like to acknowledge the assistance provided by R. Nightingale and R. Freshwater on this project.

\section{REFERENCES}

[1] IPCC, "Climate change 2013: the physical science basis. Contribution of working group I to the fifth assessment report of the intergovernmental panel on climate change," Cambridge: Cambridge University Press, 2013.

[2] G. Oberdörster et al., "Nanotoxicology: an emerging discipline evolving from studies of ultrafine particles," Environ. Health Perspect., vol. 113, pp. 823-839, 2005.

[3] J. Burkart et al., "Characterizing the performance of two optical particle counters (Grimm OPC1.108 and OPC1.109) under urban aerosol conditions," J. Aerosol Sci., vol. 41, pp. 953-962, 2010.

[4] I. Paprotny et al., "Microfabricated air-microfluidic sensor for personal monitoring of airborne particulate matter: Design, fabrication, and experimental results," in Sens. Actuators, A, vol. 201, pp. 506-516, 2013.

[5] E. Mehdizadeh, et al., "Aerosol impactor with embedded MEMS resonant mass balance for real-time particulate mass concentration monitoring," in Int. Conf. Solid State Sensors, Actuators, and Microsystems, Barcelona, 2013.

[6] S. Schmid et al., "Real-time single airborne nanoparticle detection with nanomechanical resonant filter-fiber," in Sci. Rep., vol. 3, p. 1288, 2013.

[7] H.S. Wasisto et al., "Partially integrated cantilever-based airborne nanoparticle detector for continuous carbon aerosol mass concentration monitoring," in J. Sens. Sens. Syst., vol. 4, pp. 111-123, 2015.

[8] C. Denjean et al., "Relating hygroscopicity and optical properties to chemical composition and structure of secondary organic aerosol particles generated from the ozonolysis of $\alpha$-pinene," Atmos. Chem. Phys., vol. 15, pp. 3339-3358, 2015.

[9] N.A. Hosny et al., "Direct imaging of changes in aerosol particle viscosity upon hydration and chemical aging," Chem. Sci., vol. 7, pp. 1357-1367, 2016.

[10] A. Virtanen et al., "An amorphous solid state of biogenic secondary organic aerosol particles," Nature, vol. 467, pp. 824-827, 2010.

[11] V.A. Marple and K. Willeke, "Inertial impactors: theory, design and use," in Fine Particles: Aerosol Generation, Measurement, Sampling, and Analysis, B.Y.H. Liu, Ed. New York: Academic Press, Inc., 1976, pp. 411-447.

[12] A. Prasad et al., "Simultaneous interrogation of high-Q modes in a piezoelectric-on-silicon micromechanical resonator," Sens. Actuators, A, vol. 238, pp. 207-214, 2016.

[13] A.T. Zielinski et al., "Effects of spatial sensitivity on mass sensing with bulk acoustic mode resonators," Sens. Actuators, A, vol. 236, pp. 369379, 2015.

[14] E. Diapouli et al., "Indoor and outdoor particle number and mass concentrations in Athens. Sources, sinks and variability of aerosol parameters," Aerosol Air Qual. Res., vol. 11, pp. 632-642, 2011.

[15] A. Prasad et al., "Monitoring sessile droplet evaporation on a micromechanical device," Analyst, vol. 139, pp. 5538-5546, 2014. 